\title{
USING MARKOV CHAINS TO UNDERSTAND THE SEQUENCE OF DRIVERS' GAZE TRANSITIONS DURING LANE-CHANGES IN AUTOMATED DRIVING
}

\author{
Rafael C. Gonçalves, Tyron Louw, Ruth Madigan, Natasha Merat \\ Institute for Transport Studies, University of Leeds, Leeds, UK \\ E-mail: tsrg@leeds.ac.uk
}

\begin{abstract}
Summary: This paper reports the results of a driving simulator study, which analyzed differences in drivers' raw gaze transition patterns during different stages of a lane-change maneuver, measured during manual, partially and conditionally automated driving. To understand whether the different levels of automation affected behaviour, and particularly how visual attention was distributed during a lane-change maneuver, a Markov chains approach was used to compare gaze transitions between the different information sources available in the surrounding road and cockpit environment, for each of the three drives. Results showed that drivers initiated fewer safety-related inspections (for example to the wing mirrors) during partial automation, throughout the whole lane change maneuver, possibly because they were focusing on how to the transition of control from automation. Drivers in this condition also had a higher probability of checking the system's HMI, to verify the automation's status. In contrast, during conditional automation, the lack of a need for vehicle control by the driver resulted in more gaze transitions between information sources, and fewer gazes to locations where a potential hazard could be present, when compared to manual. Finally, drivers generally only deviated their gaze towards information related to aspects of vehicle control they were responsible for, which we conclude could make them susceptible to missing hazards during both routine and safety-critical take-overs.
\end{abstract}

\section{INTRODUCTION}

It is generally agreed that the lack of a need for manual control of the vehicle, as imposed, for instance, by highly automated vehicles (AVs), removes the driver from the decision-making and control loops (Louw \& Merat, 2017), requiring them to scan the environment and acquire appropriate situation awareness (Endsley, 1995) when they are required to reengage with the driving task (Merat et al., 2018). In complex environments, and when drivers are not continually monitoring the road, but are required to resume manual control, this rebuilding of situation awareness is normally required within a short time window (Louw \& Merat, 2017). In addition, acquiring the right information at the right time requires driver attention to various parts of the road environment, and the Human Machine Interface (HMI), which should be providing drivers with the correct information, regarding automation status and, possibly, likely actions from the vehicle. In most circumstances, this information is provided to drivers at the same time, but it is not clear how drivers divide their visual attention between each of these sources, and how this diversion of visual attention is affected by the level of automation, or type of maneuver required.

When considering a lane-change maneuver, for example, to overtake a lead vehicle, drivers are required to acquire a large volume of specific information, before deciding how to act (Gipps, 1986). According to Chovan (1994), most of the accidents related to lane-changing scenarios could be avoided if drivers perform the correct safety inspection procedures. Fitch et al., (2009) 
complement this argument, by showing that drivers who do not inspect the rear-view mirrors and have long glances away from the road center have a higher probability of being involved in a crash during lane-changing tasks. Previous work on manual lane-changing behaviour has outlined the most common visual safety inspection patterns during the different stages that lead to a lane-change maneuver (Tijerina, Garrott, Stoltzfus, \& Parmer, 2005; Fitch et al., 2009; Salvucci, Liu, \& Boer, 2001). For example, Tijerina et al. (2005), have shown that prior to the execution of the maneuver, drivers generally shift their eyesight to the wing mirrors, but always shift visual attention back to the center of the road, immediately after this. When the driver initiates the maneuver, Salvucci, Liu \& Boer (2001) have shown that drivers' gaze transitions generally shift between the obstacle to be overtaken, and the destination lane. However, currently, there is a limited understanding of the drivers' distribution of eye movements during lane-changes for different levels of automated driving.

This paper provides further analysis of data from a previous study which considered drivers' gaze behaviour during automated lane-change maneuvers (Gonçalves, Louw, Madigan, Quaresma \& Merat, under review), conducted as part of the EU-funded AdaptIVe project. Here, we found that drivers engaged with different levels of vehicle automation deviate their eyesight away from the road center at similar times, as measured by Percent Road Center (PRC, see Victor et al., 2005). However, the location of drivers' visual attention was found to vary, based on the level of automation. Gaze was more spread vertically in situations where a transition of control was required (during partial automation), showing that drivers looked to the HMI placed on the dashboard. However, gaze patterns were more horizontally spread when there was no need for resumption of manual control, during conditional automation, where drivers seemed to check the maneuver execution managed by the automated lane-change. To our knowledge, this, and other research in the lane-change context (e.g. Miyajima at al., 2015), have only analyzed mean gaze fixations to different areas of interest, and drivers' average horizontal and vertical gaze dispersion. However, we argue that there is value in understanding how, and when, drivers shift their attention across the different information sources, to understand what information is used during the decision-making process required for a lane-change maneuver (Mourant \& Rockwell, 1971; Underwood, Chapman, Brockelhurst, Underwood, \& Crundall 2003).

Therefore, utilizing a Markov chains approach (Mukherjea, 1983), this study investigated how drivers distribute their attention across different parts of the road environment and vehicle, during a lane-change maneuver, and whether this behavior is different between manual, partial, and conditional, automation. This technique has been used in the past to study drivers' gaze behaviour in different situations, to understand drivers' intentions during a lane-change, and to model their scanning strategies (Underwood et al., 2003; Salvucci, Mandalia, Kuge, \& Yamamura, 2007). It can be argued that, as vehicles become more automated, this knowledge will help design more informative in-vehicle interfaces. Based on the objectives purposed above, the following research question was addressed: are there differences in the way drivers shift their visual attention focus during the stages that constitute a lane change maneuver during different levels of vehicle automation, and how is this different to when drivers are in manual control?

\section{METHODS}

Twenty-nine fully-licensed UK drivers (15 male) participated in this study. All participants had at least two years' driving experience $(\mathrm{M}=13.62, \mathrm{SD}=9.62)$ and were aged between 21 and 60 years $(\mathrm{M}=34.21, \mathrm{SD}=8.94)$. All participants were recruited through the UoLDS participant 
database and received $£ 20$ for partaking in the study. The experiment was conducted in the University of Leeds Driving Simulator (UoLDS), which is a high-fidelity, motion-based driving simulator, with a $300^{\circ}$ projection dome, containing a fully equipped Jaguar S-Type cabin is installed, with fully operational controls. Participants' eye movements were recorded using a v4.5 Seeing Machines FaceLab eye-tracker, recording at $60 \mathrm{~Hz}$.

A within-participant, 3 (Drive: manual, partial automation, conditional automation) x12 (lanechange maneuver number) repeated-measures design was used, with all participants completing the three drives (presented in a counter-balanced order). Following a short practice drive, participants completed three experimental drives. For the two automation drives, participants were instructed to maintain a speed of $70 \mathrm{mph}$ (national speed limit) and to stay in the center of the middle lane, whenever possible. In each of the three drives, participants experienced a total of 12 overtaking events (Figure 1). The overtaking events were initiated by a slower vehicle (50 $\mathrm{mph}$ ) entering the middle lane from the left lane (grey vehicle in Figure 1), blocking the path of the ego-vehicle. Participants were instructed to overtake this vehicle and to return to the middle lane, once they had passed the vehicle. This scenario was previously used in other studies from the same research group (Madigan et al., 2018; Goncalves et al., under review). The overtaking task was chosen for assessing drivers' lane-change behavior, to be consistent with previous studies on the same topic (Tijerina et al., 2005).

In the manual drive condition (MAN),

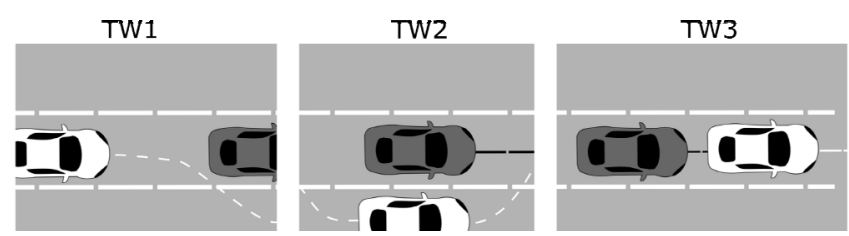

Figure 1. Representation of the scenario, showing the over-taking maneuver (TW= Time Window) drivers were in full control of the vehicle at all times. In the Partially Automated Drive (PAD) ${ }^{1}$, when engaged, the automated driving system maintained lateral and longitudinal vehicle control. However, to perform the lane-change maneuver, drivers were required to disengage the automation and perform the lane-change, by pressing a disengagement button or moving the steering wheel. They were then required to return to the middle lane and re-engage the automation as soon as it was available. In the Conditionally Automated Drive (CAD), the automation was capable of both lateral and longitudinal control of the vehicle, and performed the lane-change maneuvers, with no need for the driver to resume manual control, or to look for vehicles on the offside lane. To initiate the lane-change maneuver, drivers just had to move the indicator lever. The HMI, which was placed on the dashboard, showed the system status and an indication that a lane-change was being performed by the system.

For the analysis, the overtaking maneuver was split into three time windows (TW; see Figure. 1), guided by the work conducted by Tijerina et al. (2015) and Gipps (1986). TW1 began from when the lead vehicle entered the middle lane until the ego-vehicle exited the middle lane. In this study, we define a vehicle entering a lane when both of its front wheels cross the line dividing two lanes. TW2 began when the ego-vehicle exited the middle lane, until the point it returned to the middle lane, after overtaking the lead vehicle. TW3 began when the ego vehicle returned to the middle lane until $10 \mathrm{~s}$ after this maneuver. It must be noted that the duration of

\footnotetext{
${ }^{1}$ NB. The definitions of the levels of automation used here are the same as those adopted in our previous studies (Madigan et al., 2018; Gonçalves et al., submitted), but differ slightly from the most recent definitions adopted by SAE, (SAE, 2016), especially with respect to the hands-on requirement.
} 
TW1 and TW2 could vary for each case, based on the drivers' response. The dependent variable used for the analysis of this study was the proportion of transitions of drivers' gaze points across five AoIs (Areas of Interest). The AoIs used here were based on Carsten et al. (2012, see Figure 2 ), which were anchored around the center of the road (defined as a $6^{\circ}$ circular area centered around the mode of participants' gaze fixations during manual driving). The other four AoIs were equally divided horizontally and vertically. These comprised of the right (including the right wing-mirror); top (or rear-view mirror); left (shoulder check or left-wing mirror), and bottom (instrument cluster and system's HMI). A gaze transition was defined as the movement of drivers' eye gaze (X, Y position) from one AoI to another. Gaze-based transitions were used instead of fixation-based transitions, because short glances to the mirrors, for example, are often not detected by fixations. For the data analysis, this study opted to follow a similar approach to Underwood et al. (2003), as it allowed a direct comparison between test conditions. In this case, we used the data from the baseline drive (MAN) as the ground truth, and investigated how transitions for the other two conditions (PAD \& CAD) gaze differed from it.

The Markov chains method (Mukherjea, 1983) treats data from the gaze transitions in each driving condition, and TW, using a binomial model, in a way that every gaze transition from A to $\mathrm{B}$ has one chance $\mathrm{N}$ to happen, and is estimated based on the observed sample. $\mathrm{N}$ was calculated by the division of the number of gaze transitions from $\mathrm{A}$ to $\mathrm{B}$, by the total number of transitions that started in $\mathrm{A}$. The $\mathrm{N}$ values were used as a parameter for statistical tests, to identify where/ if specific gaze transitions (A, B) could be considered more or less probable to happen in each automation condition and TW, when compared to the manual drive. A relative value was used instead of total transitions, as the number of transitions and TW duration varied in every situation of the total sample. As the data were not normally distributed, Wilcoxon's tests were applied to measure the differences in the paired-samples of possible transitions. This paper will only report the ones with significant differences.

\section{RESULTS AND DISCUSSION}

Figure 2 and Table 1 show the results of the Wilcoxon tests, which are presented by levels of automation and TWs. Black arrows indicate that these transitions were more likely to happen, compared to what was observed during MAN, while gray dashed arrows indicate that, for both PAD and CAD, there were significantly less-frequent transitions, compared to MAN.

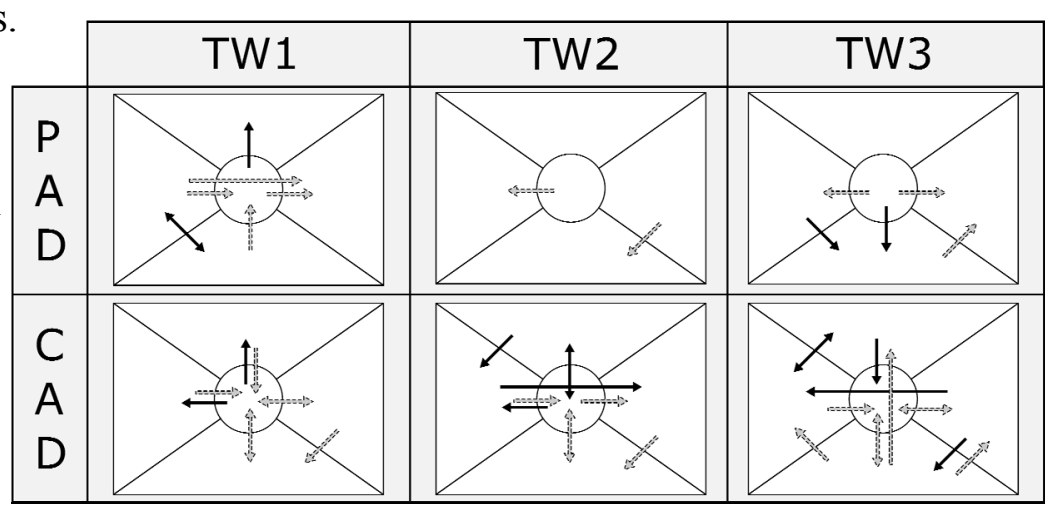

Figure 2. Representation of the differences in gaze transition frequency between AoIs for PAD and CAD compared to MAN (dotted= sig. lower than MAN; solid= sig. higher than MAN).

Figure 2 shows that PAD had a significantly higher gaze transition activity from bottom to left, and from left to bottom during TW1. A higher frequency of drivers' glances towards the bottom was also observed from the center, and the left during TW3. This pattern is in line with the change in drivers' role during the transition of control from automation to manual (TW1) and vice versa (TW3). For example, this higher frequency of gaze towards the bottom can be explained by drivers' need to look at the HMI, to check the system status information (as suggested by Louw et al., 2017a, b). These results, therefore, support the hypothesis presented in 
Gonçalves et al., (submitted), which advocates in favor of the importance of system status information on the HMI during transitions of control.

Table 1: Results of the Wilcoxon's tests on the frequency of gaze transitions between Areas of Interest.

\begin{tabular}{|c|c|c|c|c|c|c|c|c|c|c|c|c|c|c|}
\hline \multicolumn{5}{|c|}{ TW 1} & \multicolumn{5}{|c|}{ TW 2} & \multicolumn{5}{|c|}{ TW 3} \\
\hline Drive & Starting Aol & Ending Aol & $z$ & $\mathbf{P}$ & & Starting Aol & Ending Aol & $z$ & $\mathbf{P}$ & & Starting Aol & Ending Aol & $z$ & $\mathbf{P}$ \\
\hline \multirow{7}{*}{ PAD } & Center & Top & -6.189 & $<.001$ & \multirow{2}{*}{ PAD } & Center & Left & -2.119 & .034 & \multirow{5}{*}{ PAD } & Center & Left & -4.182 & $<.001$ \\
\hline & Center & Right & -4.037 & $<.001$ & & Right & Bottom & -2.121 & .033 & & Center & Bottom & -4.403 & $<.001$ \\
\hline & Left & Center & -12.199 & .028 & \multirow{10}{*}{ CAD } & Center & Top & -3.127 & .002 & & Center & Right & -2.572 & $<.001$ \\
\hline & Left & Bottom & -3.003 & .003 & & Center & Left & -3.464 & $<.001$ & & Left & Bottom & -1.42 & .016 \\
\hline & Left & Right & -2.376 & .017 & & Center & Bottom & -6.638 & $<.001$ & & Bottom & Right & -3.176 & $<.001$ \\
\hline & Bottom & Center & -2.173 & .03 & & Center & Right & -3.338 & $<.001$ & \multirow{12}{*}{ CAD } & Center & Right & -2.198 & .028 \\
\hline & Bottom & Left & -2.912 & .005 & & Top & Center & -3.495 & $<.001$ & & Top & Center & -4.818 & $<.001$ \\
\hline \multirow{9}{*}{ CAD } & Center & Top & -3.215 & $<.001$ & & Top & Left & -2.708 & .007 & & Top & Left & -2.19 & .038 \\
\hline & Center & Left & -4.534 & $<.001$ & & Top & Center & -3.383 & $<.001$ & & Left & Center & -6.532 & $<.001$ \\
\hline & Center & Bottom & -7.943 & $<.001$ & & Left & Right & -1.983 & .047 & & Left & Top & -2.229 & .026 \\
\hline & Center & Right & -3.401 & $<.001$ & & Bottom & Center & -3.401 & $<.001$ & & Bottom & Center & -4.696 & $<.001$ \\
\hline & Top & Center & -2.988 & .003 & & Right & Bottom & -2.347 & .019 & & Bottom & Top & -2 & .045 \\
\hline & Left & Center & -4.2 & $<.001$ & & & & & & & Bottom & Left & -3.536 & $<.001$ \\
\hline & Bottom & Center & -5.037 & $<.001$ & & & & & & & Bottom & Right & -2.025 & .043 \\
\hline & Right & Center & -2.536 & .011 & & & & & & & Right & Center & -2.73 & .006 \\
\hline & Right & Bottom & -3.819 & $<.001$ & & & & & & & Right & Left & -2.304 & .021 \\
\hline & & & & & & & & & & & Right & Bottom & -3.003 & .003 \\
\hline
\end{tabular}

During TW1, drivers in PAD had a significantly lower frequency of moving their eyes towards the road center (from the bottom and left) when compared to the manual drive. A lower frequency of gaze transitions towards the right from the center and left were also observed here, which suggests that drivers performed fewer glances to the mirrors to see the vehicle on the offside lane (left) and to the road center, to check the distance from the leading obstacle. During TW2, there was a lower frequency of gaze transitions from the center to the left and from the right to the bottom. Overall, in this time window, drivers' visual attention was less dispersed than during the manual drive, probably because they were checking their speedometer (bottom) and the lead vehicle (left) less often. In TW3, drivers in the PAD performed fewer mirror/shoulder checks coming from the center and bottom than the ones in MAN. According to the literature, (Tijerina et al., 2005; Salvucci, Liu, \& Boer, 2001; Fitch et al., 2009), these are common safetyrelated glance checks during the lane-change maneuver. This drop in such glances may be because of the increased workload of the driver, imposed by the transition of control, which is in line with studies reported by Crundall \& Underwood (1998) and Louw et al. (2019), who suggest that drivers have reduced scanning capabilities under high workload conditions.

It is evident that drivers presented a more scattered distribution of gaze transitions during CAD compared to MAN. During TW1, a lower frequency of gaze transitions towards the center was observed, when compared to the manual drive. The same reduction of gaze towards the center was identified in TW2 (from left and bottom) and TW3 (from left, bottom and right). Analysis of these conditions also showed a higher frequency of gaze transitions not passing through the road center during TW2 (from top to left, and from left to right) and TW3 (from top to left, from left to top, from right to left, from right to bottom). It appears that the lack of a need for vehicle control during this automation condition reduces the probability of drivers' gaze back to the road center, after attending to other AoIs. These results are in line with that of others who have shown a more dispersed gaze during automation, compared to when manual control is in place (Miyajima et al., 2015; Louw \& Merat, 2017).

Since drivers in CAD were not required to monitor the vehicle's speed or system status, assess the gap for a lane-change, or monitor the vehicle's position during TW1, a lower frequency of gaze transitions towards the right from the center and towards the bottom (from the center and the right) was observed. Since drivers still didn't need to monitor their speed in TW2, results 
showed a lower frequency of gaze to the speedometer (bottom) from the center, and the right in this TW. Fewer glances towards the destination lane, from the center,were also observed here. As drivers were not responsible for controlling most of the activities related to the lane changing task, they had no real motivation to look for information as much as they would in a manual drive. The results above support our previous assumption that drivers tend not to monitor what they are not directly in control of (Gonçalves et al., submitted; Louw \& Merat, 2017).

\section{CONCLUSION}

The aim of this study was to investigate how drivers disperse their visual attention, during manual and automated overtaking events by using a Markov Chains approach. Results indicate that, during partial automation, whenever a transition of control was required, drivers had a lower probability of performing safety-related glances, such as shifting their gaze between the side mirrors and the road center, possibly because they needed to verify the status of the system on the HMI. It has been argued that the reduction of such safety-related glances may reduce hazard detection ability (Fitch et al., 2009; Chovan, 1994), increasing the likelihood of crashes.

The results of this study also support our previous findings (Gonçalves et al., submitted), that by removing physical control and decision-making responsibility from drivers, automation reduces drivers' propensity to efficiently scan the environment and look for information that might be relevant for task execution. Therefore, removal of manual vehicle control may cause drivers to be more reliant on good system performance and suitable HMI, which, if absent, makes them less capable of responding to automation failures (Parasuraman \& Riley, 1997; Miyajima et al., 2015). This induced reliance on timely and suitable information reinforces the fact that poorly designed automated systems and related interfaces may bring with them additional and unforeseen risks to the road environment, reinforcing the ironies of automation (Bainbridge, 1981). This study demonstrates that automation reduces drivers' motivation to scan the road and vehicle environment efficiently, highlighting the need for future studies which identify how drivers' attention can be guided to the correct location, at different stages of the transition process.

\section{REFERENCES}

Bainbridge, L. (1983). Ironies of automation. In Analysis, Design and Evaluation of Man-Machine Systems 1982 (pp. 129-135).

Carsten, O., Lai, F. C., Barnard, Y., Jamson, A. H., \& Merat, N. (2012). Control task substitution in semiautomated driving: Does it matter what aspects are automated?. Human factors, 54(5), 747-761.

Chovan, J. D. (1994). Examination of lane-change crashes and potential IVHS countermeasures. National Highway Traffic Safety Administration.

Crundall, D. E., \& Underwood, G. (1998). Effects of experience and processing demands on visual information acquisition in drivers. Ergonomics, 41(4), 448-458.

Endsley, M. R. (1995). A taxonomy of situation awareness errors. Human factors in aviation operations, 3(2), 287-292.

Fitch, G. M., Lee, S. E., Klauer, S., Hankey, J., Sudweeks, J., \& Dingus, T. (2009). Analysis of lane-change crashes and near-crashes. US Department of Transportation, National Highway Traffic Safety Administration. 
Gipps, P. G. (1986). A model for the structure of lane-changing decisions. Transportation Research Part B: Methodological, 20(5), 403-414.

Goncalves R., Louw, T., Madigan, R., Merat N. (Submitted). Evaluation of gaze behaviour patterns on lane changing tasks in vehicle automation. IEEE Transactions on Human-Machine Systems.

Louw, T., \& Merat, N. (2017a). Are you in the loop? Using gaze dispersion to understand driver visual attention during vehicle automation. Transportation Research Part C: Emerging Technologies, 76, 35-50.

Louw, T., Madigan, R., Carsten, O., \& Merat, N. (2017b). Were they in the loop during automated driving? Links between visual attention and crash potential. Injury prevention, 23(4), 281-286.

Louw, T., Kuo, J., Romano, R., Radhakrishnan, V., Lenné, M., Merat, N. (2019). Engaging in NDRTs affects drivers' responses and glance patterns after silent automation failures. Transportation Research Part F: Traffic Psychology and Behaviour.

Madigan, R., Louw, T., \& Merat, N. (2018). The effect of varying levels of vehicle automation on drivers' lane changing behaviour. PloS one, 13(2), e0192190.

Merat, N., Seppelt, B., Louw, T., Engström, J., Lee, J. D., Johansson, E., Greenm C., Kitazaki, S., Monk, C., Itoh, M. \& McGehee, D. (2018). The "out-of-the-loop" concept in automated driving: Proposed definition, measures and implications. Cognition, Technology \& Work, 1-12.

Miyajima, C., Yamazaki, S., Bando, T., Hitomi, K., Terai, H., Okuda, H., \& Takeda, K. (2015, June). Analyzing driver gaze behavior and consistency of decision making during automated driving. In Intelligent Vehicles Symposium (IV), 2015 IEEE (pp. 1293-1298).

Mourant, R. R., \& Rockwell, T. H. (1972). Strategies of visual search by novice and experienced drivers. Human factors, 14(4), 325-335

Mukherjea, A. (1983). Non-Negative Matrices and Markov Chains-Seneta, E. Metrika, 30, 66-67.

Parasuraman, R., \& Riley, V. (1997). Humans and automation: Use, misuse, disuse, abuse. Human factors, 39(2), 230-253.

SAE International. (2016). Taxonomy and definitions for terms related to driving automation systems for on-road motor vehicles.

Salvucci, D. D., Liu, A., \& Boer, E. R. (2001). Control and monitoring during lane-changes. Vision in Vehicles, 9.

Salvucci, D. D., Mandalia, H. M., Kuge, N., \& Yamamura, T. (2007). Lane-change detection using a computational driver model. Human factors, 49(3), 532-542.

Tijerina, L., Garrott, W., Stoltzfus, D., \& Parmer, E. (2005). Eye glance behavior of van and passenger car drivers during lane-change decision phase. Transportation Research Record: Journal of the Transportation Research Board, (1937), 37-43.

Underwood, G., Chapman, P., Brocklehurst, N., Underwood, J., \& Crundall, D. (2003). Visual attention while driving: sequences of eye fixations made by experienced and novice drivers. aErgonomics, 46(6), 629-646.

Victor, T. (2005). Keeping eye and mind on the road (Doctoral dissertation, Acta Universitatis Upsaliensis). 